\title{
Non-Existence of Axially Symmetric Massive Scalar Fields
}

\author{
A. R. ROY and J. R. RAO \\ Department of Mathematics, Indian Institute of Technology, Kharagpur, India
}

Received March 20, 1972

\begin{abstract}
It has been observed that for the axially symmetric Einstein-Rosen metric, the stress-tensor of a scalar meson field associated with meson of rest mass $\mu$ cannot be the source term for generating gravitation. The above result also holds even when this meson field is coupled with an electromagnetic field.
\end{abstract}

\section{Introduction}

The study of scalar meson fields in general relativity has drawn the attention of many workers. Bergmann and Leipnik [1], Buchdahl [2], Bramhachary [3], Stephenson [4], Janis et al. [5], Penney [6, 7], Gautreau [8], Misra and Pandey [9] are some of the authors who have investigated various aspects of the problem.

Rao et al. [10] have recently found out exact solutions for nonstatic axially symmetric Einstein-Rosen metric when a Zeromass meson field is coupled with an electromagnetic field. A natural extension of this investigation would be the inclusion of the mass of the meson field. To this end, we have taken up the problem of interacting gravitational and massive (nonzero-rest-mass) scalar meson fields in Section 2, and in Section 3 we have studied the more general case when the massive scalar field is coupled with an electromagnetic field. In both the cases it has been observed that the stress-tensor of the massive scalar meson field cannot be the source term for generating gravitation. This result excludes the possibility of any further extension of our previous work [10].

\section{Axially Symmetric Massive Scalar Field}

We consider the axially symmetric Einstein-Rosen metric

$$
d s^{2}=e^{2 \alpha-2 \beta}\left(d t^{2}-d \varrho^{2}\right)-\varrho^{2} e^{-2 \beta} d \phi^{2}-e^{2 \beta} d z^{2},
$$

where $\alpha$ and $\beta$ are functions of $\varrho$ and $t$ only and $\varrho, \phi, z, t$ correspond respectively to $x^{1}, x^{2}, x^{3}, x^{4}$ coordinates. 
The general relativistic field equations for regions of spacetime containing matter are

$$
G_{i j} \equiv R_{\imath \jmath}-\frac{1}{2} R g_{i j}=-\kappa T_{\imath \jmath},
$$

where $\kappa\left(=\frac{8 \pi G}{c^{4}}\right)$ is the gravitational constant.

In this section we consider the solutions of the field Eqs. (2.2) when $T_{i j}$ represents the stress-energy tensor for a scalar meson field associated with a meson of rest mass $\mu$, where $V$ satisfies the scalar wave equation

$$
g^{i j} V_{; i j}+\mu^{2} V=0
$$

the semicolon denoting covariant differentiation.

Taking now the stress-tensor of the massive scalar menson field (Corson [11]) as

$$
T_{i j}=\frac{1}{4 \pi}\left[V_{, i} V_{, j}-\frac{1}{2} g_{i j}\left(V_{, s} V^{, s}-\mu^{2} V^{2}\right)\right],
$$

the field Eqs. (2.2) become

$$
G_{i j} \equiv R_{i j}-\frac{1}{2} R g_{i j}=-\frac{\kappa}{4 \pi}\left[V_{, i} V_{, j}-\frac{1}{2} g_{i j}\left(V_{, s} V^{, s}-\mu^{2} V^{2}\right)\right],
$$

where a comma denotes partial differentiation and small latin letters take the values $1,2,3,4$.

With the nonvanishing components of the Ricci tensor (see Eq. (11) of $[10])$, the field Eqs. (2.5) are

$$
\begin{aligned}
& G_{11} \equiv \beta_{1}^{2}+\beta_{4}^{2}-\frac{\alpha_{1}}{\varrho} \\
&=-\frac{\kappa}{4 \pi}\left[\left(V_{, 1}\right)^{2}-\frac{1}{2} g_{11}\left(V_{, 1} V^{, 1}+V_{, 4} V^{, 4}-\mu^{2} V^{2}\right)\right], \\
& G_{22} \equiv \varrho^{2} e^{-2 \alpha}\left(-\alpha_{11}+\alpha_{44}-\beta_{1}^{2}+\beta_{4}^{2}\right) \\
&=-\frac{\kappa}{4 \pi}\left[-\frac{1}{2} g_{22}\left(V_{, 1} V^{, 1}+V_{, 4} V^{, 4}-\mu^{2} V^{2}\right)\right], \\
& G_{33} \equiv e^{4 \beta-4 \alpha}\left(-2 \beta_{11}-2 \beta_{44}-\alpha_{11}+\alpha_{44}-\beta_{1}^{2}+\beta_{4}^{2}+\frac{2 \beta_{1}}{\varrho}\right) \\
&=-\frac{\kappa}{4 \pi}\left[-\frac{1}{2} g_{33}\left(V_{, 1} V^{, 1}+V_{, 4} V^{, 4}-\mu^{2} V^{2}\right)\right], \\
& G_{44} \equiv \beta_{1}^{2}+\beta_{4}^{2}-\frac{\alpha_{1}}{\varrho} \\
&=-\frac{\kappa}{4 \pi}\left[\left(V_{, 4}\right)^{2}-\frac{1}{2} g_{44}\left(V_{, 1} V^{, 1}+V_{, 4} V^{, 4}-\mu^{2} V^{2}\right)\right],
\end{aligned}
$$


and

$$
G_{14} \equiv 2 \beta_{1} \beta_{4}-\frac{\alpha_{4}}{\varrho}=-\frac{\kappa}{4 \pi} V_{, 1} V_{, 4},
$$

where the axial symmetry assumed implies that the scalar potential $V$ shares the same symmetry as $\alpha$ and $\beta$, as a consequence of which

$$
V, 2=0, \quad V_{3}=0 \text {. }
$$

Thus the axially symmetric massive scalar field will be completely determined by the Eqs. (2.6)-(2.10) and (2.3).

The Eqs. (2.6) and (2.9) give us

$$
g^{11} \mu^{2} V^{2}=0
$$

which implies that $\mu=0$. Hence, we conclude that for the axially symmetric Einstein-Rosen metric (2.1) there cannot exist any massive scalar field. Physically we can interpret this as showing that massive scalar fields cannot be the source term for generating axially symmetric gravitational field described by (2.1).

\section{Axially Symmetric Coupled Electromagnetic and Massive Scalar Field}

In this section we consider the region of the spacetime to consist of a sourcefree electromagnetic field, in addition to the massive scalar field, satisfying the Maxwell's equations

and

$$
\begin{gathered}
F_{i j}=A_{i, j}-A_{j, i} \\
F^{i j} ; j=0,
\end{gathered}
$$

where $F_{i j}$ is the electromagnetic field tensor and $A_{i}$ the electromagnetic four potential.

Thus, in this case $T_{i j}$ consists of two parts viz., stress-tensor corresponding to the electromagnetic field given by

$$
\frac{1}{4 \pi}\left[-F_{i s} F_{j}^{s}+\frac{1}{4} g_{i j} F_{s p} F^{s p}\right]
$$

and that corresponding to the massive scalar field already defined by (2.4).

Hence, the field Eqs. (2.2) for this case are

$$
\begin{aligned}
G_{i j} \equiv R_{i j}-\frac{1}{2} R g_{i j}= & -\frac{\kappa}{4 \pi}\left[-F_{i s} F_{j}^{s}+\frac{1}{4} g_{i j} F_{s p} F^{s p}\right] \\
& -\frac{\kappa}{4 \pi}\left[V_{, i} V_{, j}-\frac{1}{2} g_{i j}\left(V_{, s} V^{, s}-\mu^{2} V^{2}\right)\right] .
\end{aligned}
$$

Thus the Eqs. (2.3), (3.1), (3.2) and (3.4) will determine the field completely. 
For the metric (2.1) we have the relation

i.e.

$$
R_{2}^{2}+R_{3}^{3}=0 \text {, }
$$

$$
G_{1}^{1}+G_{4}^{4}=0 \text {, }
$$

which in view of (3.4) implies

$$
g^{11} g^{44}\left(F_{14}\right)^{2}-g^{22} g^{33}\left(F_{23}\right)^{2}=0 .
$$

Since $g^{11}, g^{22}, g^{33}$ are all negative and $g^{44}$ is positive (3.5) will hold iff

$$
F_{14}=0, \quad F_{23}=0 \text {. }
$$

Substituting the values of the Ricci tensors and using (3.6), the field Eqs. (3.4) read as

$$
\begin{aligned}
& G_{11} \equiv \beta_{4}^{2}+\beta_{1}^{2}-\frac{\alpha_{1}}{\varrho}=-\frac{\kappa}{4 \pi}\left\{-F_{12} F_{1}^{2}-F_{13} F_{1}^{3}\right. \\
& \left.+\frac{1}{2} g_{11}\left(F_{12} F^{12}+F_{13} F^{13}+F_{24} F^{24}+F_{34} F^{34}\right)\right\} \\
& -\frac{\kappa}{8 \pi}\left[\left(V_{, 1}\right)^{2}+\left(V_{.4}\right)^{2}+g_{11} \mu^{2} V^{2}\right] \text {, } \\
& G_{22} \equiv \varrho^{2} e^{-2 \alpha}\left(-\alpha_{11}+\alpha_{44}-\beta_{1}^{2}+\beta_{4}^{2}\right)=-\frac{\kappa}{4 \pi}\left\{-F_{21} F_{2}^{1}-F_{24} F_{2}^{4}\right. \\
& \left.+\frac{1}{2} g_{22}\left(F_{12} F^{12}+F_{13} F^{13}+F_{24} F^{24}+F_{34} F^{34}\right)\right\} \\
& -\frac{\kappa}{4 \pi}\left\{-\frac{1}{2} g_{22}\left(V_{, 1} V^{, 1}+V_{, 4} V^{, 4}-\mu^{2} V^{2}\right)\right\} \text {, } \\
& G_{33} \equiv e^{4 \beta-4 \alpha}\left(-2 \beta_{11}-2 \beta_{44}-\alpha_{11}+\alpha_{44}-\beta_{1}^{2}+\beta_{4}^{2}+\frac{2 \beta_{1}}{\varrho}\right) \\
& =-\frac{\kappa}{4 \pi}\left\{-F_{31} F_{3}^{1}-F_{34} F_{3}^{4}\right. \\
& \left.+\frac{1}{2} g_{33}\left(F_{12} F^{12}+F_{13} F^{13}+F_{24} F^{24}+F_{34} F^{34}\right)\right\} \\
& -\frac{\kappa}{4 \pi}\left\{-\frac{1}{2} g_{33}\left(V_{, 1} V^{, 1}+V_{, 4} V^{, 4}-\mu^{2} V^{2}\right)\right\} \\
& G_{44} \equiv \beta_{4}^{2}+\beta_{1}^{2}-\frac{\alpha_{1}}{\varrho}=-\frac{\kappa}{4 \pi}\left\{-F_{42} F_{4}^{2}-F_{43} F_{4}^{3}\right. \\
& \left.+\frac{1}{2} g_{44}\left(F_{12} F^{12}+F_{13} F^{13}+F_{24} F^{24}+F_{34} F^{34}\right)\right\} \\
& -\frac{\kappa}{8 \pi}\left[\left(V_{, 1}\right)^{2}+\left(V_{.4}\right)^{2}-g_{11} \mu^{2} V^{2}\right] \text {, } \\
& G_{14} \equiv 2 \beta_{1} \beta_{4}-\frac{\alpha_{4}}{\varrho}=-\frac{\kappa}{4 \pi}\left\{\left(-F_{12} F_{4}^{2}-F_{13} F_{4}^{3}\right)\right\}-\frac{\kappa}{4 \pi} V_{, 1} V_{, 4} \text {. }
\end{aligned}
$$


Now multiplying (3.7) by $g^{11}$ and (3.10) by $g^{44}$ and noting $g^{11}=-g^{44}$, we get

$$
\mu=0 \text {. }
$$

Hence, there cannot exist any solution for the coupled electromagnetic and massive scalar field for the metric (2.1).

We may mention in conclusion that the above results are also true when the field characterized by (2.1) is assumed to be static.

\title{
References
}

1. Bergmann, O., Leipnik, R.: Phys. Rev. 107, 1157 (1957).

2. Buchdahl, H.: Phys. Rev. 115, 1325 (1959).

3. Bramhachary, R. L.: Progr. Theoret. Phys. 23, 749 (1960).

4. Stephenson, G.: Proc. Cambridge Phil. Soc. 58, part 3, 521 (1962).

5. Janis, A.I., Newman, E., Winicour, J.: Phys. Letters 20, 878 (1968).

6. Penney, R.: Phys. Rev. 174, 1578 (1968).

7. - Phys. Rev. 182, 1383 (1969).

8. Gautreau, R.: Nuovo Cimento B, Series X, 62, 360 (1969).

9. Misra, R. M., Pandey, D. B.: Commun. math. Phys. 20, 324 (1971).

10. Rao,J.R., Roy, A. R., Tiwari, R. N.: Ann. Phys. 69, no. 1, 473 (1972).

11. Corson,E.M.: Introduction to tensors, spinors and relativistic wave equations, p. 96. London and Glasgow: Blackie 1953.

A. R. Roy

J. R. Rao

Department of Mathematics

Indian Institute of Technology

Kharagpur, India

\section{Erratum}

\section{Theory of Monomer-Dimer Systems}

\author{
O. J. Heilmann and E. H. Lieb
}

Commun. math. Phys. 25, 190-232(1972)

The legends to the figures were inadvertently omitted; they should read as follows:

Fig. 1. The sign variation of $Q(G ; x)$ for $N(G)=7$ as $x$ goes from $-\infty$ to $+\infty$.

Fig. 2. Two graphs for which $Q(G ; x)$ has two identical zeros. (a) gives two zeros at $x=0$ independent of the edge weights. In (b) if the weights on all four vertical edges are $a^{2}$ there will be double zeros at $x= \pm a$. 